\title{
Epilepsy versus non-epileptic attack disorder: A diagnostic and therapeutic challenge
}

\author{
Catherine Smith ${ }^{*}$, Jason Ramtahal
}

South Devon Healthcare NHS Foundation Trust, Torquay, UK;

*Corresponding Author: catherine.smith31@nhs.net, jason.ramtahal@nhs.net

Received 26 January 2013; revised 28 February 2013; accepted 4 March 2013

\begin{abstract}
Epilepsy and non-epileptic attack disorder (NEAD) share a vast number of clinical features, however the aetiology and management are very different. Video-EEG is the gold standard diagnostic tool and relies on the occurrence of seizure activity during assessment to make a diagnosis. Added complexity arises from the coexistence of epilepsy and NEAD, occurring in a significant proportion of patients. Comprehensive assessment and investigation is therefore required to prevent gross mistreatment in this diagnostically difficult subgroup. We present a case of NEAD with co-existing epilepsy and the challenges that this may present in clinical practice.
\end{abstract}

Keywords: Epilepsy; Non-Epileptic Attack Disorder; NEAD; Seizure; Video-EEG

\section{INTRODUCTION}

A seizure may be defined as a sudden attack of disease most commonly referring to the physical manifestations resulting from abnormal electrical discharges in the brain [1]. However, up to $40 \%$ of patients referred to specialist centres with supposed refractory epilepsy are found to have no organic cause for their seizures and a diagnosis of non-epileptic attack disorder (NEAD) is made [2-4]. Clinically, NEAD is characterised by involuntary episodes of disruptive behaviour, thinking, emotion or sensation that resemble epileptic seizures but with persistent absence of epileptiform activity on electroencephalogram (EEG) [5]. Seizures of NEAD commonly resemble frontal lobe seizures due to the behavioural aspects of their nature, however patients and witnesses report a diversity of manifestations including phenomena traditionally associated with epilepsy [6,7]. Video-EEG is the gold standard investigatory tool and previous to its implementation, diagnosis relied heavily on clinical judgement [8]. It is widely recognised that epilepsy and NEAD may coexist. There is much dispute over the estimated prevalence of comorbidity, with reports of up to $50 \%$ of patients with NEAD having concurrent epilepsy [9]. The risk of misdiagnosis is significant and the paroxysmal nature of seizure activity in both epilepsy and NEAD increases the difficulty in making a diagnosis despite the use of videoEEG assessment.

This report describes a case of coexistent NEAD and epilepsy diagnosed on video-EEG in a patient with unrecognised long-standing paroxysmal seizure activity and a previously normal EEG. The report aims to explore the diagnostic techniques and treatment methods of a patient presenting with seizures and to highlight the potential for mismanagement in this group of patients.

\section{CLINICAL CASE}

A 20-year-old woman was admitted to hospital having had a vacant episode with subsequent amnesia and bizarre behaviour the following day. Absent episodes, as reported by her mother, have occurred since the age of 7 or 8 . The patient would have vacant spells during conversation, occurring 2 to 3 times per year. An EEG performed 5 years previously showed no abnormality. Three years ago, whilst pregnant, her mother reported that the attacks were getting worse. She would wake from sleep and walk out into the road. Her partner witnessed her sitting up in bed during the night, tapping her head and reports having to stop her beating herself up during sleep. The majority of attacks occurred at night and included the patient shouting out. She has no recollection of the attacks. Since childhood, she has suffered with night terrors, characterised by sudden waking from sleep, feeling angry or upset or in a state of heightened arousal. Past medical history also includes asthma, irritable bowel syndrome and alpha thalassaemia trait. At the age of 12, she saw a counsellor due to deliberate self-harm (DSH) to her arms by scratching over a period of 6 months. She attributes this behaviour to a challenging family environment and reports resolution with counselling. At the 
time of initial assessment all medications had been stopped. Her father had complex partial seizures as a child and has a diagnosis of epilepsy characterised by petit mal seizures. Her mother described the seizures as resembling her father's. She has one paternal grandparent and one maternal grandparent with epilepsy. She has one brother with cerebral palsy and another with progressive muscular problems. Her uncle has schizophrenia. She is a nonsmoker, rarely drinks alcohol and works as a residential home officer for children with special needs. She lives with her mother, father, two brothers and 2-year-old son.

Neurology consultation following hospital admission revealed a normal neurological examination and clinically, a sleep disorder rather than a seizure disorder was suspected. A CT scan of her head was performed and no abnormality was found. In addition, the patient underwent sleep studies and video-EEG telemetry. This demonstrated multiple clinical events reported by the patient and nursing staff such as blurred vision, tingling, urinary incontinence and absent episodes with no associated EEG abnormalities. However, the study also demonstrated four nocturnal clinical events, unreported by the patient and nursing staff, characterised primarily by spontaneous arousal from stage II sleep, vocalisation and repetitive movements. Concurrent electroclinical findings provided strong evidence that these episodes were epileptic in origin. The episodes were stereotypical and suggestive of frontal lobe seizures. On receipt of these results, an MRI scan of the brain was requested looking for a frontal focus of her seizures. No structural abnormality was detected. The patient was started on $25 \mathrm{mg}$ of Lamotrigine once a day, increasing to an eventual dose of $50 \mathrm{mg}$ twice daily and subsequently her sleep has improved. At most recent follow-up (9 months post-initiation of Lamotrigine), she remained well and her night time sleep was well maintained (it is thought that her frontal lobe seizures may have contributed to poor night time sleep).

\section{DISCUSSION}

The present case study describes a patient with seizure activity clinically suggestive of NEAD though strictly indistinguishable from epileptic seizures, particularly those of frontal lobe origin. The patient had a previously normal EEG and several risk factors for NEAD, including vast familial ill health. On further investigation, both epileptic and non-epileptic activity was demonstrated on videoEEG.

Seizures are extremely common with an incidence of up to 1 per 1000 per year [10]. Aetiologies are multiple and to discern the origin of a seizure is of utmost importance with regards to management [11]. Identification of epileptiform seizures is important due to the potential benefit of AEDs and equally the risk of iatrogenic misuse of such drugs in non-epileptic seizures. There are many ways to categorise epileptic seizures, for example they can be divided by location within the brain such as frontal or temporal lobe seizures, or by clinical presentation such as tonic-clonic or absence seizures. Classification, and therefore precise diagnosis, is highly important in order to tailor treatment to the type of seizure and the individual patient [12].

Similarly, seizures originating from NEAD can manifest in a variety of ways from behavioural disturbance to motor, sensory or autonomic phenomena and clinically they can mimic all types of epileptic seizure [7,11]. Due to the clinical similarities between NEAD and epileptic seizures, much difficulty has been reported in reaching a definitive diagnosis [7]. Added complexity arises from the coexistence of epileptic and non-epileptic seizures in an individual patient such as in the present case. Comorbidity poses the risk of making a diagnosis based on what is thought to be accurate and thorough investigation, to find that a second aetiology is present and responsible for symptoms previously attributed to the original diagnosis. Multiple studies have found a high prevalence of co-morbidity. The proportion of patients with NEAD found to have concurrent epilepsy is quoted between $10 \%$ and $60 \%$ whereas a minority of the epileptic population are thought to have coexisting NEAD, with a prevalence of $5 \%-20 \%[2,4]$. One argument for the high rate of co-morbidity in patients with NEAD is the idea of sick-role behaviour whereby patients subconsciously adopt similar behavioural symptoms characteristic of their childhood epilepsy, manifesting as non-epileptic seizures. The pathophysiology of functional neurological symptoms is not yet fully understood. It is best described using a multi-factorial model of aetiology, incorporating numerous biological, psychological and social elements. One model suggests that childhood experience, such as child ill health and associated parental attitude, could play a crucial role in the development of "somatiform" coping mechanisms later in life [13].

Video-EEG is primarily used to locate the anatomical origin of a patient's seizures, to determine candidates suitable for surgery and to differentiate between epileptic and non-epileptic seizures [14]. Patients undergo video telemetry as an inpatient and are typically analysed for a period between days and weeks. This manner of investigation is extremely important regarding the diagnosis of coexistent seizure aetiologies, however care must be taken with such a definitive diagnostic tool to ensure adequate assessment so that additional diagnoses are not missed. Equally cost, both to the patient and the NHS, must be compared with the benefit of long-term video telemetry, and research into the necessary length of assessment would be useful in evaluating the cost-benefit ratio [15].

The aetiology of NEAD is complex and there are many 
associated risk factors to suggest the diagnosis [11]. The present case study demonstrates a female patient of typical age, with a family history of ill health (including several members with epilepsy) and a personal history of DSH, night terrors and a self-proclaimed challenging family environment. Some studies suggest that ill health of a sibling or other close family member can contribute to the development of NEAD in a patient who subconsciously requires parental attention that may be lacking within the family unit. There is no evidence that these episodes are factitious and it is important to approach the topic with care when discussing this with patients and their families $[5,16]$. There is a further theory suggesting that subconscious mimicking behaviour of patients with epileptic family members could contribute to the manifestations of NEAD. This further supports the theory that symptoms of somatisation may develop as a result of early learning experiences in childhood. Interestingly, the seizures reported in the present case study closely resembled those of the patient's father. It has been recognised that adverse family dynamics could play a significant role in the development of NEAD. Childhood abuse, whether sexual, physical or emotional, is considered a risk factor, however one study found that an inadequate family environment was more common than sexual or physical abuse in these patients [16]. Concurrent psychiatric illness is also a well-recognised risk factor for NEAD, with one study reporting a prevalence of nearly 75\% (mood disorders and personality disorders are most commonly associated) [11]. Aetiologically, NEAD is a psychiatric condition requiring psychotherapeutic intervention. According to ICD 10, "pseudoseizures" are a clinical feature of dissociative (conversion) disorder; therefore thorough psychiatric assessment is needed to distinguish whether the diagnosis is NEAD as a sole entity or whether the seizures are a symptom of another psychiatric disorder [17]. Symptomatology is complex, whereby the origin is psychological yet the expression neurological, thus emphasising that collaboration between specialties is paramount [11]. Delayed or missed diagnosis of NEAD can result in a myriad of adverse consequences. The most obvious is inadequate treatment, whether due to the iatrogenic use of AEDs or the delay of psychotherapeutic intervention. Side effects of AEDs include hypersensitivity, teratogenicity, gastrointestinal disturbance, and electrolyte imbalance among others [18]. Evidence has shown the use of certain AEDs and their withdrawal to be associated with autonomic nervous system dysfunction and increased vulnerability to cardiac arrhythmias. Both of which could contribute to sudden unexpected death [19]. Admittedly, these studies were conducted in patients with epilepsy and similar research in patients with non-epileptic seizures is limited. Further investigation in this patient subgroup could pro- vide useful information, potentially adding breadth to this argument. Delay in initiating psychological management has been linked to poor prognosis in patients with NEAD, with multiple studies reporting an average delay of approximately 7 to 8 years in making a diagnosis [20, 21]. The importance of prompt initiation of treatment is reiterated in two studies that reported the cessation of seizures in approximately one third of all patients 6 - 12 months following video-EEG diagnosis [22,23].

\section{CONCLUSIONS}

1) The coexistence of NEAD and epilepsy creates great diagnostic difficulty for clinicians.

2) This emphasises the importance of thorough and accurate investigation, including detailed history and examination and appropriate use of video-EEG.

3) The aetiology of NEAD is multi-factorial, with a strong psychological basis in most cases.

4) Management requires collaboration between both psychological and neurological teams in order to provide good clinical care for patients with NEAD.

5) Delayed or missed diagnoses could lead to adverse consequences such as the iatrogenic use of AEDs or the under-treatment of patients with NEAD and/or epilepsy.

In conclusion the comparable presentations of epilepsy and NEAD create a diagnostic and therapeutic challenge thus requiring comprehensive assessment by the appropriate specialty teams using gold standard investigation techniques in order to prevent gross mismanagement of this subgroup of patients.

\section{REFERENCES}

[1] Dorland, W. (2003) Dorland's illustrated medical dictionary. Saunders, Philadelphia.

[2] Smith, D., Defalla, B.A. and Chadwick, D.W. (1999) The misdiagnosis of epilepsy and the management of refractory epilepsy in a specialist clinic. Quarterly Journal of Medicine, 92, 15-23. doi:10.1093/qjmed/92.1.15

[3] Benbadis, S.R. and Allen, H.W. (2000) An estimate of the prevalence of psychogenic non-epileptic seizures. Seizure, 9, 280-281. doi:10.1053/seiz.2000.0409

[4] Jones, S.G., O’Brien, T.J., Adams, S.J., et al. (2010) Clinical characteristics and outcome in patients with psychogenic nonepileptic seizures. Psychosomatic Medicine, 72, 487-497. doi:10.1097/PSY.0b013e3181d96550

[5] LaFrance Jr., W.C. and Devinsky, O. (2004) Treatment of nonepileptic seizures: Historical perspectives and future directions. Epilepsia, 45, 15-21. doi:10.1111/j.0013-9580.2004.452002.x

[6] Reuber, M., Jamnadas-Khoda, J., Broadhurst, M., et al. (2011) Psychogenic nonepileptic seizure manifestations reported by patients and witnesses. Epilepsia, 52, 20282035. doi:10.1111/j.1528-1167.2011.03162.x 
[7] Rowan, A.J. (2000) Diagnosis of non-epileptic seizures. In: Gates, J.R., Rowan, A.J., Eds., Non-Epileptic Seizures, 2nd Edition, Butterworth-Heinemann, Boston, 15-30.

[8] Lesser, R.P. (1986) Psychogenic seizures. Psychosomatics, 27, 823-829. doi:10.1016/S0033-3182(86)72587-5

[9] Marchetti, R.L., Kurcgant, D., Gallucci-Neto, J., Von Bismark, M.A. and Fiore, L.A. (2010) Epilepsy in patients with psychogenic non-epileptic seizures. Arquivos de Neuro-Psiquiatria, 68, 168-173. doi:10.1590/S0004-282X2010000200003

[10] Hauser, W.A. and Beghi, E. (2008) First seizure definitions and worldwide incidence and mortality. Epilepsia, 49, 8-12. doi:10.1111/j.1528-1167.2008.01443.x

[11] Alsaadi, T.M. and Marquez, A.V. (2005) Psychogenic nonepileptic seizures. American Family Physician, 72, 849-856.

[12] Glauser, T., Ben-Menachem, E., Bourgeois, B., et al. (2006) ILAE treatment guidelines: evidence-based analysis of antiepileptic drug efficacy and effectiveness as initial monotherapy for epileptic seizures and syndromes. Epilepsia, 47, 1094-1120. doi:10.1111/j.1528-1167.2006.00585.x

[13] Twamley, E.W. and Bortz, J.J. (1999) Nonepileptic seizures: Neuropsychological mechanisms. BNI Quarterly, 15, 20-26.

[14] Ghougassian, D.F., d'Souza, W., Cook, M.J. and O’Brien, T.J. (2004) Evaluating the utility of inpatient video-EEG monitoring. Epilepsia, 45, 928-932. doi:10.1111/j.0013-9580.2004.51003.x

[15] Szaflarski, J.P., Hughes, C., Szaflarski, M., et al. (2003) Quality of life in psychogenic nonepileptic seizures. Epilepsia, 44, 236-242. doi:10.1046/j.1528-1157.2003.35302.x
[16] Vincentiis, S., Valente, K.D., Thomé-Souza, S., Kuczinsky, E., Fiorea, L.A. and Negrãoa, N. (2006) Risk factors for psychogenic nonepileptic seizures in children and adolescents with epilepsy. Epilepsy \& Behavior, 8, 294298. doi:10.1016/j.yebeh.2005.08.014

[17] World Health Organization. The ICD-10 classification of mental and behavioural disorders. Clinical descriptions and diagnostic guidelines. http://www.who.int/classifications/icd/en/bluebook.pdf

[18] Epilepsy Society (2012) Table of anti-epileptic drugs used in adults. http://www.epilepsysociety.org.uk/AboutEpilepsy/Treatm ent/Antiepilepticdrugsusedinadults

[19] Kennebäck, G., Ericson, M., Tomson, T. and Bergfeldt, L. (1997) Changes in arrhythmia profile and heart rate variability during abrupt withdrawal of antiepileptic drugs. Implications for sudden death. Seizure, 6, 369-375. doi:10.1016/S1059-1311(97)80036-2

[20] Mellers, J.D.C. (2004) The approach to patients with "non-epileptic seizures”. Postgraduate Medical Journal, 13, 146-155.

[21] Reuber, M., Fernández, G., Bauer, J., Helmstaedter, C. and Elger, C.E. (2004) Diagnostic delay in psychogenic nonepileptic seizures. Neurology, 58, 493-495. doi:10.1212/WNL.58.3.493

[22] Lempert, T. and Schmidt, D. (1990) Natural history and outcome of psychogenic seizures: A clinical study in 50 patients. Journal of Neurology, 237, 35-38. doi:10.1007/BF00319665

[23] Kanner, A.M., Parra, J., Frey, M., Stebbins, G., PierreLouis, S. and Iriarte, J. (1999) Psychiatric and neurologic predictors of psychogenic pseudoseizure outcome. Neurology, 53, 933-938. doi:10.1212/WNL.53.5.933 